\title{
Trends in Self-Reported Sitting Time by Physical Activity Levels Among US Adults, NHANES 2007/2008-2017/2018
}

\author{
Emily N. Ussery, Geoffrey P. Whitfield, Janet E. Fulton, Deborah A. Galuska, Charles E. Matthews, \\ Peter T. Katzmarzyk, and Susan A. Carlson
}

\begin{abstract}
Background: High levels of sedentary behavior and physical inactivity increase the risk of premature mortality and several chronic diseases. Monitoring national trends and correlates of sedentary behavior and physical inactivity can help identify patterns of risk in the population over time. Methods: The authors used self-reported data from the National Health and Nutrition Examination Surveys (2007/2008-2017/2018) to estimate trends in US adults' mean daily sitting time, overall, and stratified by levels of leisure-time and multidomain physical activity, and in the joint prevalence of high sitting time ( $>8 \mathrm{~h} / \mathrm{d})$ and physical inactivity. Trends were tested using orthogonal polynomial contrasts. Results: Overall, mean daily sitting time increased by 19 minutes from $2007 / 2008(332 \mathrm{~min} / \mathrm{d})$ to $2017 / 2018(351 \mathrm{~min} / \mathrm{d})\left(P_{\text {linear }}<.05 ; P_{\text {quadratic }}<.05\right)$. The highest point estimate occurred in 2013/2014 (426 min/d), with a decreasing trend observed after this point $\left(P_{\text {linear }}<.05\right)$. Similar trends were observed across physical activity levels and domains, with one exception: an overall linear increase was not observed among sufficiently active adults. The mean daily sitting time was lowest among highly active adults compared with less active adults when using the multidomain physical activity measure. Conclusions: Sitting time among adults increased over the study period but decreased in recent years.
\end{abstract}

Keywords: sedentary behavior, surveillance, population health

The Physical Activity Guidelines for Americans, second edition ("Guidelines") includes guidance for adults to move more and sit less throughout the day and states that adults who sit less and do any amount of moderate- to vigorous-intensity physical activity gain some health benefits. ${ }^{1}$ Regular physical activity is associated with a range of health benefits, including reduced risk of many chronic diseases and premature all-cause mortality. ${ }^{2} \mathrm{~A}$ substantial body of evidence also shows a strong relationship between time spent in sedentary behavior, often assessed as daily sitting time, and adverse health outcomes, including an increased risk of premature cardiovascular disease and all-cause mortality. ${ }^{2}$ Both high sedentary behavior and physical inactivity have negative health effects, and the risk of premature mortality is particularly elevated when they occur together. ${ }^{2,3}$ Growing evidence also suggests the mortality risks associated with sedentary time are attenuated at higher levels of moderate- to vigorous-intensity physical activity. ${ }^{2,3}$ Monitoring population trends in the combination of sedentary behavior and physical activity can help identify patterns of risk in the population over time.

The National Health and Nutrition Examination Survey (NHANES) is the only US surveillance system that regularly monitors self-reported sitting time and physical activity. ${ }^{4}$ Using data from NHANES, studies have found a significant increase of

Ussery, Whitfield, Fulton, Galuska, and Carlson are with the Division of Nutrition, Physical Activity, and Obesity, National Center for Chronic Disease Prevention and Health Promotion, Centers for Disease Control and Prevention, Atlanta, GA, USA. Matthews is with the Division of Cancer Epidemiology and Genetics, National Cancer Institute, Rockville, MD, USA. Katzmarzyk is with the Pennington Biomedical Research Center, Louisiana State University, Baton Rouge, LA, USA. Carlson is also with the Division of Population Health, National Center for Chronic Disease Prevention and Health Promotion, Centers for Disease Control and Prevention, Atlanta, GA, USA. Ussery (yzv4@cdc.gov) is corresponding author. about 40 minutes per day in self-reported sitting time among US adults from 2007/2008 to 2015/2016.5,6 Physical activity levels have also increased over the past several years ${ }^{7,8}$; for example, a recent analysis of NHANES multidomain activity found $68 \%$ of adults met the aerobic guideline in 2017/2018, up from $64 \%$ in 2007/2008. ${ }^{7}$ However, to our knowledge, no studies to date have examined whether trends in sitting time vary by physical activity levels. A better understanding of how sitting time varies by levels of physical activity in leisure time, the most commonly assessed physical activity domain in the United States, ${ }^{4}$ and in multiple domains can provide insight into the population risk levels of these behaviors and inform strategies to help reduce sitting time and increase physical activity. For example, if increases in sitting time were primarily occurring among adults who are also physically inactive, this would be particularly concerning given current evidence demonstrating that the health risks associated with high levels of sedentary behavior are exacerbated by low levels of physical activity.

In addition, understanding how commonly high sitting time and physical inactivity co-occur in the adult population, how this joint prevalence is changing over time, and the demographic correlates of these joint behaviors could help establish the current magnitude of risk in the population and identify high-risk groups. One study found that $11 \%$ of US adults reported sitting for $>8$ hours per day and engaging in no leisure-time physical activity in 2015/ 2016. 9 Another study found a temporal increase in the joint prevalence of sitting for $>6$ hours per day and not adhering to the aerobic physical activity guideline of $\geq 150$ minutes of moderate-intensity equivalent physical activity per week across multiple domains (eg, leisure-time, occupational/household, and transportation) from 2007/2008 to 2015/2016. ${ }^{5}$ Studies that have examined correlates of combined high sitting time and physical inactivity are limited, but evidence exists to suggest older age and higher body 
mass index (BMI) are independent risk factors for each of these behaviors. ${ }^{10-14}$ The present study updates and extends previous findings by examining recent trends in the joint prevalence of high sitting time and physical inactivity and the demographic correlates of these combined high-risk behaviors to inform future strategies.

Using nationally representative data from 2007/2008 to 2017/ 2018, we had 3 specific objectives. First, we estimate trends in selfreported sitting time among US adults overall and by physical activity levels. Second, we estimate trends in the combined prevalence of high sitting time and physical inactivity. Third, we examine demographic characteristics associated with the joint behaviors. To determine if findings vary when considering additional domains of physical activity beyond leisure time, we use 2 measures of physical activity throughout: (1) leisure-time physical activity only and (2) multidomain (ie, leisure time, occupational/ household, and transportation-related) physical activity.

\section{Methods}

\section{Data Source and Sample}

The data from adult respondents $(\geq 18$ y old $)$ in 6 cycles of NHANES were analyzed: 2007/2008 $(n=6228), 2009 / 2010$ $(\mathrm{n}=6527), 2011 / 2012(\mathrm{n}=5864), 2013 / 2014(\mathrm{n}=6113), 2015 /$ $2016(\mathrm{n}=5992)$, and 2017/2018 $(\mathrm{n}=5856)$. NHANES combines in-person interviews with physical examinations to assess the health and nutritional status of US youth and adults. It uses a stratified, multistage, probability cluster sample of the civilian, noninstitutionalized population, and data are released in 2-year increments. Additional information on the sampling methodology and data collection procedures are available on the NHANES website (https://www.cdc.gov/nchs/nhanes/). From 2007/2008 through 2017/2018, interview response rates ranged from a low of $51.9 \%(2017 / 2018)$ to a high of $79.4 \%$ (2009/2010). For this analysis, individuals were excluded if they were missing data on sitting time $(n=217)$, physical activity $(n=104)$, demographic characteristics $(n=66)$, or measured BMI $(n=1890)$. The final analytic sample consisted of 34,303 adults.

\section{Measures}

Sitting Time. In 2007/2008, daily sitting time was assessed with the following question: "How much time do you usually spend sitting or reclining on a typical day?" The respondents were asked to include the amount of waking time spent "sitting or reclining at work, at home, or at school, including time spent sitting at a desk, sitting with friends, traveling in a car, bus, or train, reading, playing cards, watching television, or using a computer." In subsequent cycles, a similar question was asked without the phrase "or reclining." Daily sitting time was categorized as $<4$ hours per day, 4 to $<6$ hours per day, 6 to 8 hours per day, and $>8$ hours per day ("high sitting time"), consistent with categories used in other large epidemiologic studies. . $^{3,9,15}$

Physical Activity. In all cycles, occupational/household physical activity, active transportation, and leisure-time physical activity were assessed separately using questions about the frequency (in days per week) and duration (in minutes per day) of activities done for at least 10 minutes continuously in a typical week. Vigorousintensity activities were defined as those "that require hard physical effort and cause large increases in breathing or heart rate," and moderate-intensity activities as those "that require moderate physical effort and cause small increases in breathing or heart rate."
First, the respondents separately reported vigorous-intensity and moderate-intensity activities done as part of "paid or unpaid work, household chores, and yard work" (ie, occupational/household activity). Second, the respondents reported the time spent "walking or using a bicycle for at least 10 minutes continuously to get to and from places" (ie, transportation-related activity); these activities were considered moderate intensity per the analytic notes provided for the NHANES physical activity questionnaire (https:/wwwn.cdc. gov/Nchs/Nhanes/2017-2018/PAQ_J.htm\#Analytic_Notes). Third, the respondents separately reported vigorous intensity and moderate intensity "sports, fitness and recreational activities" (ie, leisure-time activity). Within each domain, the frequency and duration of moderate- and vigorous-intensity activity were multiplied to calculate the total weekly volume. Per guidelines, the volume of vigorousintensity physical activity was multiplied by 2 and added to moderate-intensity physical activity to create moderate-intensity equivalent minutes. ${ }^{1}$ Two variables were created, representing the volume of leisure-time physical activity and the volume of multidomain physical activity (leisure time, occupational/household, and transportation-related). For each, the respondents were categorized based on moderate-intensity equivalent minutes as inactive (<10 $\mathrm{min} / \mathrm{wk})$, insufficiently active (10-149 $\mathrm{min} / \mathrm{wk})$, sufficiently active (150-300 $\mathrm{min} / \mathrm{wk})$, or highly active (>300 $\mathrm{min} / \mathrm{wk})$ according to current aerobic guidelines. ${ }^{1}$

Demographic Characteristics. The demographic characteristics assessed in the interview included sex, age, race, ethnicity, and highest level of educational attainment. Age was categorized as 18-34 years, 35-44 years, 45-64 years, and $\geq 65$ years. Race and ethnicity were combined into a single variable and categorized as non-Hispanic white (white), non-Hispanic black (black), Hispanic, and non-Hispanic other or multiple races. Education level was categorized as less than high school, high school graduate, some college, and college graduate or above. The respondents reported their employment status during the past week, and each was categorized as employed ("working at a job or business" or "with a job or business but not at work"), not employed ("not working at a job or business" due to reasons other than retirement, or "looking for work"), or retired. BMI, calculated using weight and height measured during the in-person examination, was categorized as under or normal weight $\left(<25.0 \mathrm{~kg} / \mathrm{m}^{2}\right)$, overweight $(25.0$ to $\left.<30.0 \mathrm{~kg} / \mathrm{m}^{2}\right)$, or having obesity $\left(\geq 30.0 \mathrm{~kg} / \mathrm{m}^{2}\right) .{ }^{16}$

\section{Data Analysis}

The weighted descriptive characteristics were calculated for each NHANES cycle. For each cycle, the mean daily sitting time (in minutes per day) was estimated with $95 \%$ confidence interval (CI) overall and stratified by level of leisure time only and multidomain physical activity. Within cycles, pairwise differences in mean sitting time by physical activity levels were tested using adjusted Wald tests. Absolute differences in mean sitting time from 2007/ 2008 to $2017 / 2018$ were calculated, and linear and quadratic trends over time were tested using linear regression with orthogonal polynomial contrasts. Given a significant overall quadratic trend and the observed inflection point in 2013/2014, trends before and after this time point (segment 1, 2007/2008 to 2013/2014; segment $2,2013 / 2014$ to $2017 / 2018$ ) were also examined. For each cycle, the joint prevalence of high sitting time and physical inactivity was estimated, and trends were tested using logistic regression with orthogonal polynomial contrasts. For the most recent survey cycle (2017/2018), the demographic differences in the joint prevalence of high sitting time and physical inactivity were evaluated using 
logistic regression; crude and adjusted odds ratios with $95 \%$ CIs were calculated. The odds ratios were adjusted for sex, age, race/ ethnicity, education level, employment status, and BMI status. For all analyses, the significance level was set at $P<.05$. The analyses were conducted in Stata (version 13.1; StataCorp, College Station, TX) using survey commands to account for sampling weights and survey design characteristics.

\section{Results}

Sample-weighted demographic characteristics, physical activity levels, and categories of sitting time are summarized in Table 1 for each survey cycle. In each cycle, just under one-half of the adults were physically inactive when considering only leisure-time physical activity, and just over one-fifth were physically inactive when considering multidomain physical activity. In 2007/2008, $33.6 \%$ reported sitting for $<4$ hours per day and $18 \%$ reported sitting for $>8$ hours per day, compared with $26.9 \%$ and $19.7 \%$ in 2017/2018, respectively.

\section{Overall Trends in Sitting Time}

Overall, the sitting time increased by 19 minutes from 332 minutes per day $(5.5 \mathrm{~h} / \mathrm{d})$ in $2007 / 2008$ to 351 minutes per day $(5.9 \mathrm{~h} / \mathrm{d})$ in $2017 / 2018$. The shape of the trend was both significantly linear and quadratic $\left(P_{\text {linear }}<.05 ; P_{\text {quadratic }}<.05\right)$, thus demonstrating nonlinear variation in addition to an overall increase over time (Figure 1). The highest point estimate occurred in 2013/2014 (426 min/d). Two segments with significant linear trends were observed before and after 2013/2014 (change from 2007/2008 to 2013/2014: $94.4 \mathrm{~min}$ increase $\left[P_{\text {linear }}<.05 ; P_{\text {quadratic }}<.05\right]$, change from 2013/2014 to 2017/2018: 75.4 min decrease $\left.\left[P_{\text {linear }}<005\right]\right)$.

\section{Trends and Differences in Sitting Time by Physical Activity Levels}

Across all levels of leisure time only and multidomain physical activity, from $2007 / 2008$ to $2017 / 2018$, the mean daily sitting time exhibited a positive linear trend with a negative quadratic trend $\left(P_{\text {linear }}<.05 ; P_{\text {quadratic }}<.05\right)$ with one exception: linear trends were not significant among the sufficiently active group regardless of the domain examined (Table 2). The highest point estimate for the mean daily sitting time occurred in 2013/2014 across all levels of leisure-time only and multidomain physical activity (Figure 2). When examining segments before and after 2013/2014, there were significant linear increases of between 56.2 and 119.5 minutes per day before this point $\left(P_{\text {linear }}<.05\right.$ for all subgroups $)$ and significant linear decreases of between 50.3 and 99.8 minutes per day after this point $\left(P_{\text {linear }}<.05\right.$ for all subgroups $)$. For 3 subgroups, a significant quadratic trend was observed between 2007/2008 and 2013/2014 in addition to the linear increase $\left(P_{\text {quadratic }}<.05\right)$ : inactive adults in both leisure time only and multidomain physical activity, and insufficiently active adults in multidomain physical activity. In each survey cycle, the mean sitting time was lowest among the highly active adults (eg, 2017/2018: $323.2 \mathrm{~min} / \mathrm{d}$; 95\% CI, 304.3-342.2) compared with the inactive $(396.0 \mathrm{~min} / \mathrm{d} ; 95 \% \mathrm{CI}$, $376.3-415.7)$, insufficiently active $(369.8 \mathrm{~min} / \mathrm{d}$; $95 \%$ CI, 342.2397.3), and sufficiently active (390.7 $\mathrm{min} / \mathrm{d}$; 95\% CI, 363.8-417.6) adults when using the multidomain physical activity measure (Table 2). When using leisure-time physical activity, the comparison between highly active and other categories was less consistent across the survey cycles.

\section{Joint Prevalence of High Sitting Time and Physical Inactivity}

The joint prevalence of high sitting time and leisure-time physical inactivity increased from $8.2 \%$ in $2007 / 2008$ to $8.7 \%$ in $2017 / 2018$ $\left(P_{\text {linear }}<.05 ; P_{\text {quadratic }}<.05 ;\right.$ Figure 3$)$. The joint prevalence of high sitting time and multidomain physical inactivity was lower in magnitude at each survey cycle than comparable estimates using only leisure-time inactivity (eg, 5.8\% in 2007/2008 and 5.5\% in 2017/2018), and a significant quadratic trend was observed with no significant linear trend $\left(P_{\text {quadratic }}<.05\right)$. The highest joint prevalence estimate was observed in $2013 / 2014$ for both leisure-time inactivity (14.4\%) and multidomain inactivity (9.5\%); for both measures, a linear increasing trend with a significant deviation was observed before this point $\left(P_{\text {linear }}<.05 ; P_{\text {quadratic }}<.05\right)$, with a significant linear decreasing trend after this point $\left(P_{\text {linear }}<.05\right)$.

\section{Demographic Differences in Joint Prevalence}

Regardless of whether leisure time only or multidomain physical activity was included, the unadjusted odds of reporting both high sitting time and physical inactivity were greater among adults aged $\geq 65$ years compared with 18-34 years, greater among adults with obesity compared with under/normal weight adults, and lower among Hispanic compared with white adults (Table 3). The unadjusted odds of high sitting time and physical inactivity were greater among retired adults compared with employed adults only when multidomain physical activity was included. When the analyses were adjusted for demographic characteristics, the results were similar, with 2 exceptions: the odds were no longer significant for Hispanic compared with white adults and for retired compared with employed adults when using the multidomain physical activity measure.

\section{Discussion}

From $2007 / 2008$ to $2017 / 2018$, daily sitting time increased overall by 19 minutes per day among US adults, although more recent data points suggest sitting time has decreased over the last 4-5 years, showing some improvement. Similar trends were observed among inactive, insufficiently active, and highly active adults, regardless of whether only leisure-time activity or multidomain physical activity was considered. Trends in jointly reported high sitting time and physical inactivity followed a similar pattern, with the highest prevalence in 2013/2014 and decreasing trends through 2017/2018. Nationwide efforts to implement evidence-based programs and policies to reduce sedentary behavior where adults live, learn, work, and play may help ensure the observed improvement continues.

This study updates previous trend analyses of self-reported sitting time $^{5,6}$ with the most recent NHANES data cycle. While previous studies documented an increase of about 40 minutes of daily sitting time between 2007/2008 and 2015/2016,5,6 the inclusion of the most recent data in our study resulted in a smaller overall increase. We also documented a significant linear decrease in the mean sitting time since its peak in $2013 / 2014$. When examining these trends by physical activity levels categorized using 2 different physical activity measures, the findings were similar to those for the overall sample. However, highly active adults using the multidomain physical activity measure had a consistently lower mean sitting time compared with those who were less active. The magnitude of decreases in sitting time since 2013/2014 across physical activity levels is encouraging, given the evidence of elevated mortality risks associated with higher sitting 


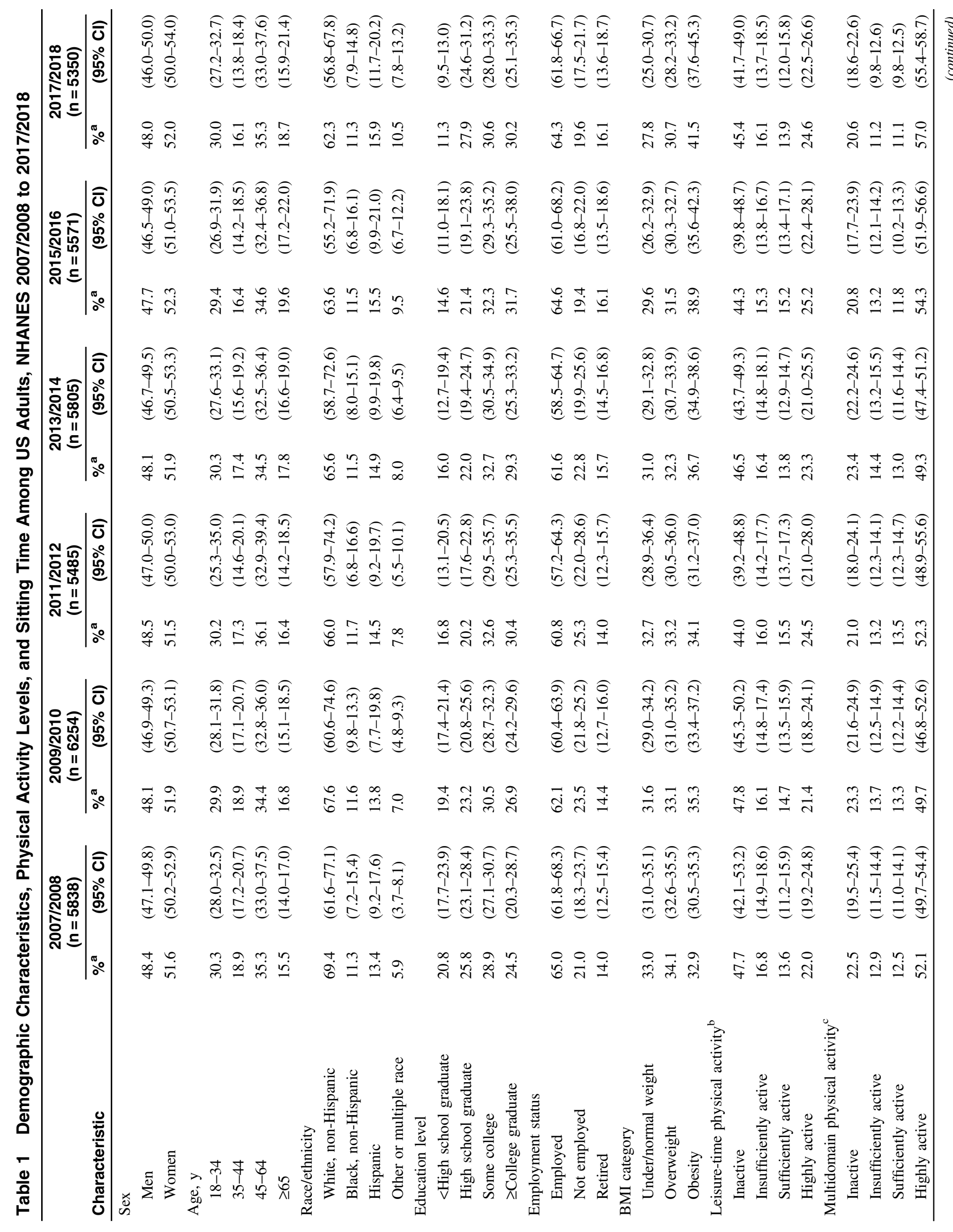




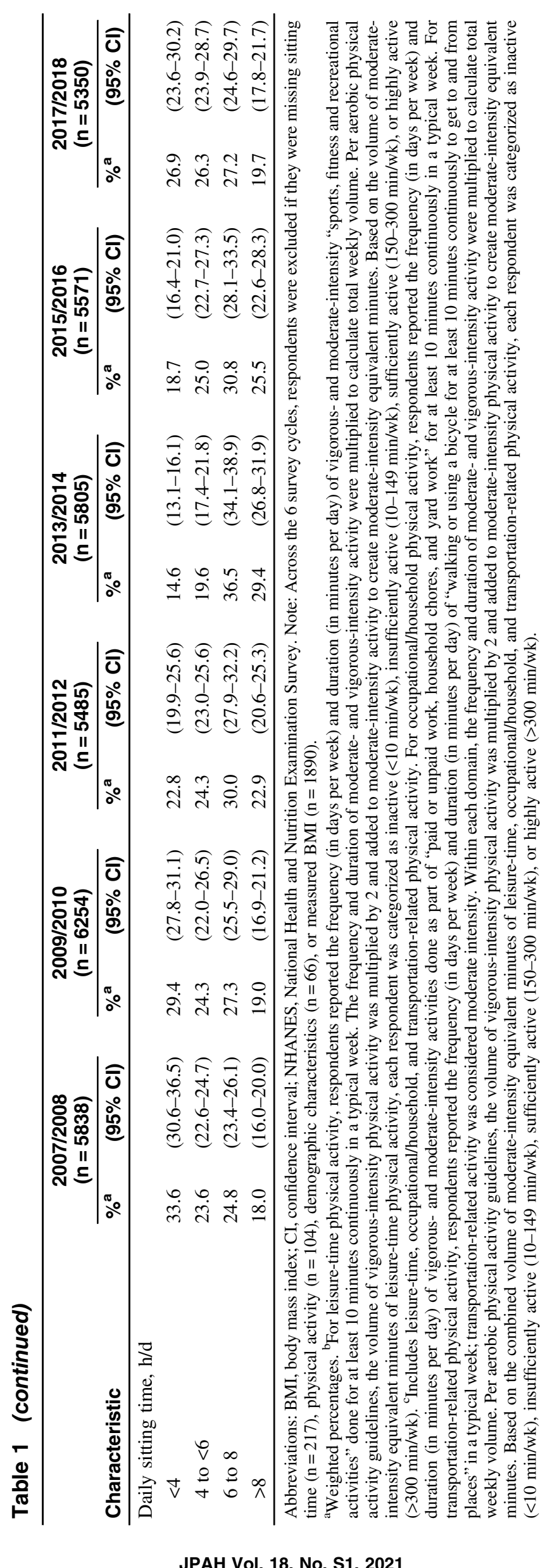




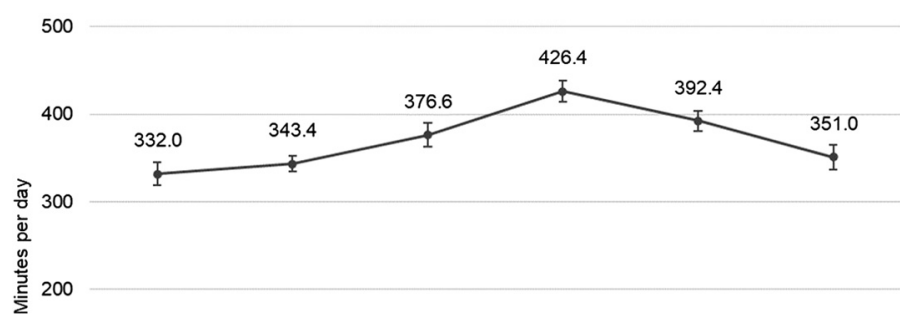

100

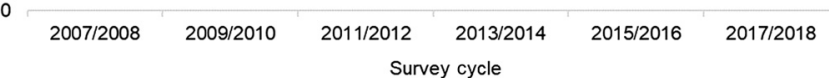

Figure 1 - Trends in mean daily sitting time among US adults, NHANES 2007/2008 to 2017/2018. Note. Error bars indicate 95\% CIs. CI indicates confidence interval; NHANES, National Health and Nutrition Examination Survey. time, particularly among those with lower levels of physical activity. ${ }^{3,15}$ Future work may focus on continuing these trends, especially among adults who are physically inactive or do not meet the aerobic activity guideline.

Similar to our findings on mean sitting time, the joint prevalence of high sitting time and physical inactivity also peaked in 2013/2014, with progressively lower prevalence in the 2 cycles since, regardless of which physical activity measure was used. The guidelines recommend that adults move more and sit less, and physical activity in any domain is considered to be beneficial for health. ${ }^{1}$ Therefore, the distinction between leisure time only and multidomain inactivity is important when calculating the joint prevalence. The joint prevalence of high sitting and physical inactivity may be overestimated when evaluating only physical activity in leisure time, because some people may be active through nonleisure pursuits. Our findings thus reinforce the importance of multidomain assessment of physical activity for understanding the true patterns of risk associated with sitting time and low levels of physical activity in the population. Moreover, NHANES assesses

Table 2 Trends in Mean Daily Sitting Time Among US Adults Overall and By Physical Activity Level, NHANES $2007 / 2008$ to $2017 / 2018$

\begin{tabular}{|c|c|c|c|c|c|c|c|c|c|c|c|c|}
\hline \multirow[b]{3}{*}{ Physical Activity Level } & \multicolumn{6}{|c|}{ Daily sitting time } & \multicolumn{6}{|c|}{ Change } \\
\hline & \multicolumn{2}{|c|}{$2007 / 2008$} & \multicolumn{2}{|c|}{$2013 / 2014$} & \multicolumn{2}{|c|}{$2017 / 2018$} & \multicolumn{2}{|c|}{ Segment $1^{a}$} & \multicolumn{2}{|c|}{ Segment $2^{a}$} & \multicolumn{2}{|c|}{ Overall $^{a}$} \\
\hline & $\begin{array}{c}\text { Mean, } \\
\text { min }\end{array}$ & $(95 \% \mathrm{Cl})$ & $\begin{array}{l}\text { Mean, } \\
\text { min }\end{array}$ & $(95 \% \mathrm{Cl})$ & $\begin{array}{c}\text { Mean, } \\
\text { min }\end{array}$ & $(95 \% \mathrm{Cl})$ & $\begin{array}{l}\text { Abs, } \\
\text { min }\end{array}$ & Trend & $\begin{array}{l}\text { Abs, } \\
\text { min }\end{array}$ & Trend & $\begin{array}{c}\text { Abs, } \\
\text { min }\end{array}$ & Trend \\
\hline Overall & 332.0 & $\begin{array}{c}(318.8- \\
345.2)\end{array}$ & 426.4 & $\begin{array}{c}(414.5- \\
438.4)\end{array}$ & 351.0 & $\begin{array}{c}(336.7- \\
365.2)\end{array}$ & 94.4 & $\mathrm{~L}, \mathrm{Q}$ & -75.4 & $\mathrm{~L}$ & 19.0 & $\mathrm{~L}, \mathrm{Q}$ \\
\hline \multicolumn{13}{|c|}{ Leisure-time physical activity ${ }^{\mathrm{b}}$} \\
\hline Inactive & 324.6 & $\begin{array}{l}(309.3- \\
340.0)^{\mathrm{A}}\end{array}$ & 444.1 & $\begin{array}{c}(429.3- \\
458.8)\end{array}$ & 352.5 & $\begin{array}{l}(334.0- \\
371.0)^{\mathrm{A}}\end{array}$ & 119.5 & $\mathrm{~L}, \mathrm{Q}$ & -91.6 & $\mathrm{~L}$ & 27.9 & $\mathrm{~L}, \mathrm{Q}$ \\
\hline Insufficiently active & 346.7 & $\begin{array}{c}(318.5- \\
375.0)^{\mathrm{A}, \mathrm{B}}\end{array}$ & 427.0 & $\begin{array}{l}(405.3- \\
448.7)^{\mathrm{A}}\end{array}$ & 349.6 & $\begin{array}{l}(325.1- \\
374.1)^{\mathrm{A}}\end{array}$ & 80.3 & $\mathrm{~L}$ & -77.4 & $\mathrm{~L}$ & 2.9 & $\mathrm{~L}, \mathrm{Q}$ \\
\hline Sufficiently active & 359.3 & $\begin{array}{l}(328.8- \\
389.8)^{\mathrm{B}}\end{array}$ & 415.5 & $\begin{array}{l}(400.0- \\
431.0)^{\mathrm{A}}\end{array}$ & 356.2 & $\begin{array}{l}(330.5- \\
381.8)^{\mathrm{A}}\end{array}$ & 56.2 & $\mathrm{~L}$ & -59.3 & $\mathrm{~L}$ & -3.1 & Q \\
\hline Highly active & 320.0 & $\begin{array}{l}(305.3- \\
334.8)^{\mathrm{A}}\end{array}$ & 397.3 & $\begin{array}{c}(381.5- \\
413.0)\end{array}$ & 346.1 & $\begin{array}{l}(321.4- \\
370.8)^{\mathrm{A}}\end{array}$ & $\mathbf{7 7 . 3}$ & $\mathrm{L}$ & -51.2 & $\mathrm{~L}$ & 26.1 & $\mathrm{~L}, \mathrm{Q}$ \\
\hline \multicolumn{13}{|c|}{ Multidomain physical activity } \\
\hline Inactive & 384.6 & $\begin{array}{l}(366.5- \\
402.7)^{\mathrm{A}}\end{array}$ & 489.4 & $\begin{array}{l}(469.4- \\
509.4)^{\mathrm{A}}\end{array}$ & 396.0 & $\begin{array}{l}(376.3- \\
415.7)^{\mathrm{A}}\end{array}$ & 104.8 & $\mathrm{~L}, \mathrm{Q}$ & -93.4 & $\mathrm{~L}$ & 11.4 & $\mathrm{~L}, \mathrm{Q}$ \\
\hline Insufficiently active & 371.4 & $\begin{array}{l}(343.5- \\
399.3)^{\mathrm{A}}\end{array}$ & 469.6 & $\begin{array}{l}(442.1- \\
497.1)^{\mathrm{A}}\end{array}$ & 369.8 & $\begin{array}{l}(342.2- \\
397.3)^{\mathrm{A}}\end{array}$ & 98.2 & $\mathrm{~L}, \mathrm{Q}$ & -99.8 & $\mathrm{~L}$ & -1.6 & $\mathrm{~L}, \mathrm{Q}$ \\
\hline Sufficiently active & 380.9 & $\begin{array}{l}(354.4- \\
407.4)^{\mathrm{A}}\end{array}$ & 441.0 & $\begin{array}{c}(426.2- \\
455.9)\end{array}$ & 390.7 & $\begin{array}{l}(363.8- \\
417.6)^{\mathrm{A}}\end{array}$ & 60.1 & $\mathrm{~L}$ & -50.3 & $\mathrm{~L}$ & 9.8 & Q \\
\hline Highly active & 287.8 & $\begin{array}{c}(274.8- \\
300.7)\end{array}$ & 380.1 & $\begin{array}{c}(366.0- \\
394.2)\end{array}$ & 323.2 & $\begin{array}{c}(304.3- \\
342.2)\end{array}$ & 92.3 & $\mathrm{~L}$ & -56.9 & $\mathrm{~L}$ & 35.4 & $\mathrm{~L}, \mathrm{Q}$ \\
\hline
\end{tabular}

Abbreviations: Abs, absolute change; CI, confidence interval; L, significant linear trend; NHANES, National Health and Nutrition Examination Survey; Q, significant quadratic trend. Note: Uppercase letters denote results of pairwise comparisons of predicted marginal means. Estimates sharing a letter are not significantly different at the .05 level. Estimates shown in bold represent a significant absolute change in mean daily sitting time $(P<.05)$ over the specified time period.

${ }^{a}$ Segment 1, 2007/2008 to 2013/2014; segment 2, 2013/2014 to 2017/2018; overall, 2007/2008 to 2017/2018. ${ }^{b}$ For leisure-time physical activity, respondents reported the frequency (in days per week) and duration (in minutes per day) of vigorous- and moderate-intensity "sports, fitness and recreational activities" done for at least 10 minutes continuously in a typical week. The frequency and duration of moderate- and vigorous-intensity activity were multiplied to calculate total weekly volume. Per aerobic physical activity guidelines, the volume of vigorous-intensity physical activity was multiplied by 2 and added to moderate-intensity activity to create moderate-intensity equivalent minutes. Based on the volume of moderate-intensity equivalent minutes of leisure-time physical activity, each respondent was categorized as inactive ( $<10$ min/ wk), insufficiently active (10-149 $\mathrm{min} / \mathrm{wk})$, sufficiently active (150-300 $\mathrm{min} / \mathrm{wk})$, or highly active ( $>300 \mathrm{~min} / \mathrm{wk}){ }^{\circ}$ Includes leisure-time, occupational/household, and transportation-related physical activity. For occupational/household physical activity, respondents reported the frequency (in days per week) and duration (in minutes per day) of vigorous- and moderate-intensity activities done as part of "paid or unpaid work, household chores, and yard work" for at least 10 minutes continuously in a typical week. For transportation-related physical activity, respondents reported the frequency (in days per week) and duration (in minutes per day) of "walking or using a bicycle for at least 10 minutes continuously to get to and from places" in a typical week; transportation-related activity was considered moderate intensity. Within each domain, the frequency and duration of moderate- and vigorous-intensity activity were multiplied to calculate total weekly volume. Per aerobic physical activity guidelines, the volume of vigorous-intensity physical activity was multiplied by 2 and added to moderate-intensity physical activity to create moderate-intensity equivalent minutes. Based on the combined volume of moderate-intensity equivalent minutes of leisure-time, occupational/household, and transportation-related physical activity, each respondent was categorized as inactive ( $<10 \mathrm{~min} / \mathrm{wk})$, insufficiently active (10-149 $\mathrm{min} / \mathrm{wk})$, sufficiently active (150-300 min/wk), or highly active ( $>300 \mathrm{~min} / \mathrm{wk})$. 

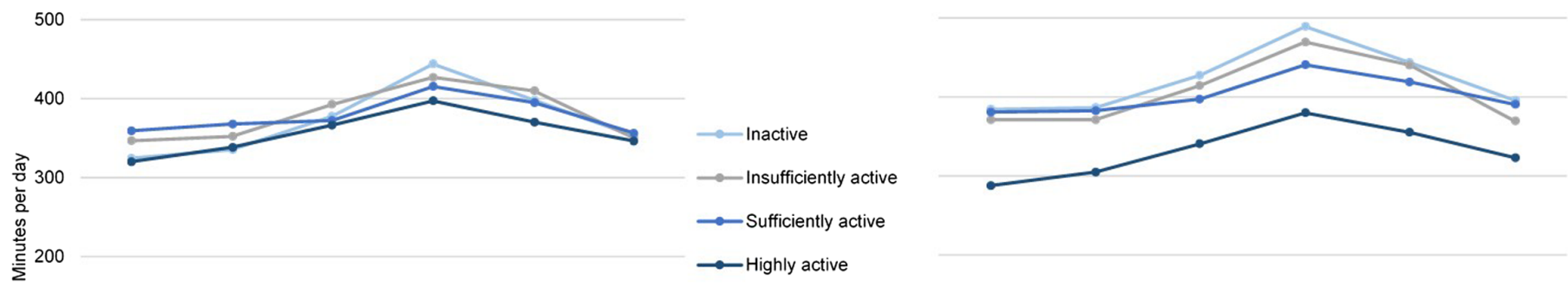

100

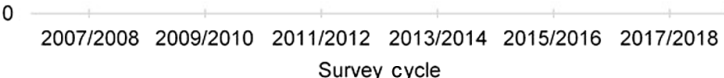

$2007 / 2008 \quad 2009 / 2010 \quad 2011 / 2012 \quad 2013 / 2014 \quad 2015 / 2016 \quad 2017 / 2018$ Survey cycle

Figure 2 - Trends in mean daily sitting time among US adults by levels of leisure-time and multidomain physical activity, NHANES 2007/2008 to 2017/2018. *For leisure-time physical activity, respondents reported the frequency (in days per week) and duration (in minutes per day) of vigorous- and moderate-intensity "sports, fitness and recreational activities" done for at least 10 minutes continuously in a typical week. The frequency and duration of moderate- and vigorous-intensity activity were multiplied to calculate total weekly volume. Per aerobic physical activity guidelines, the volume of vigorous-intensity physical activity was multiplied by 2 and added to moderate-intensity activity to create moderate-intensity equivalent minutes. Based on the volume of moderate-intensity equivalent minutes of leisure-time physical activity, each respondent was categorized as inactive ( $<10$ min/wk), insufficiently active (10-149 min/wk), sufficiently active (150-300 min/wk), or highly active ( $>300 \mathrm{~min} / \mathrm{wk}){ }^{\dagger}$ Leisure-time, occupational/household, and transportation-related physical activity. For occupational/household physical activity, respondents reported the frequency (in days per week) and duration (in minutes per day) of vigorous- and moderate-intensity activities done as part of "paid or unpaid work, household chores, and yard work" for at least 10 minutes continuously in a typical week. For transportation-related physical activity, respondents reported the frequency (in days per week) and duration (in minutes per day) of "walking or using a bicycle for at least 10 minutes continuously to get to and from places" in a typical week; transportation-related activity was considered moderate intensity. Within each domain, the frequency and duration of moderate- and vigorous-intensity activity were multiplied to calculate total weekly volume. Per aerobic physical activity guidelines, the volume of vigorous-intensity physical activity was multiplied by 2 and added to moderate-intensity physical activity to create moderate-intensity equivalent minutes. Based on the combined volume of moderate-intensity equivalent minutes of leisure-time, occupational/household, and transportation-related physical activity, each respondent was categorized as inactive $(<10 \mathrm{~min} / \mathrm{wk})$, insufficiently active (10-149 min/wk), sufficiently active (150-300 min/wk), or highly active ( $>300 \mathrm{~min} / \mathrm{wk})$. NHANES indicates National Health and Nutrition Examination Survey.

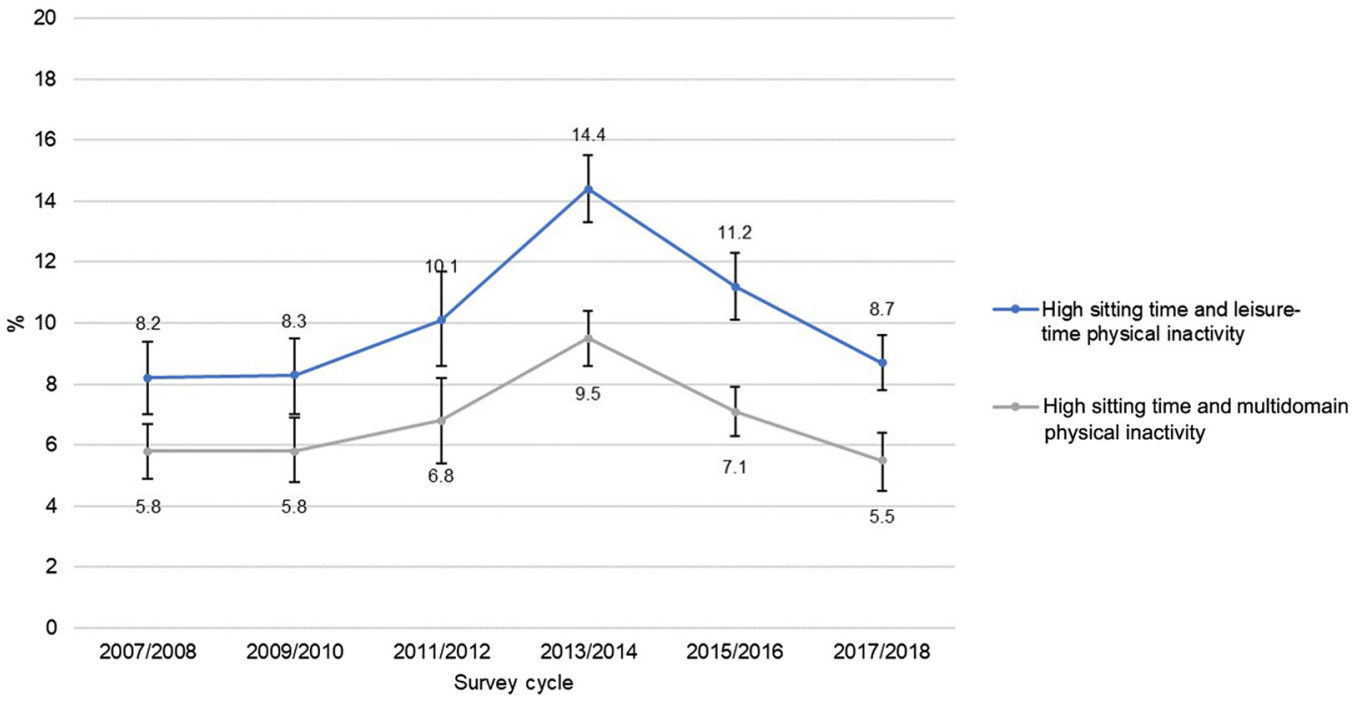

Figure 3 - Trends in the joint prevalence of high sitting time ( $>8 \mathrm{~h} / \mathrm{d})$ and physical inactivity among US adults by domain of physical activity, NHANES 2007/2008 to 2017/2018. Note. The blue line represents the percentage who reported both sitting for more than 8 hours per day and engaging in $<10$ minutes per week of leisure-time physical activity. The gray line represents the percentage who reported both sitting for $>8$ hours per day and engaging in $<10$ minutes per week of multidomain (leisure-time, occupational/household, or transportation-related) physical activity. Error bars represent 95\% CIs. From 2007/2008 to 2017/2018, a significant positive linear trend and negative quadratic trend $(P<.05)$ were observed for the joint prevalence of high sitting time and leisure-time physical inactivity. From 2007/2008 to 2017/2018, a significant negative quadratic trend $(P<.05)$ was observed for the joint prevalence of high sitting time and multidomain physical inactivity, with no significant linear trend. CI indicates confidence interval; NHANES, National Health and Nutrition Examination Survey. 


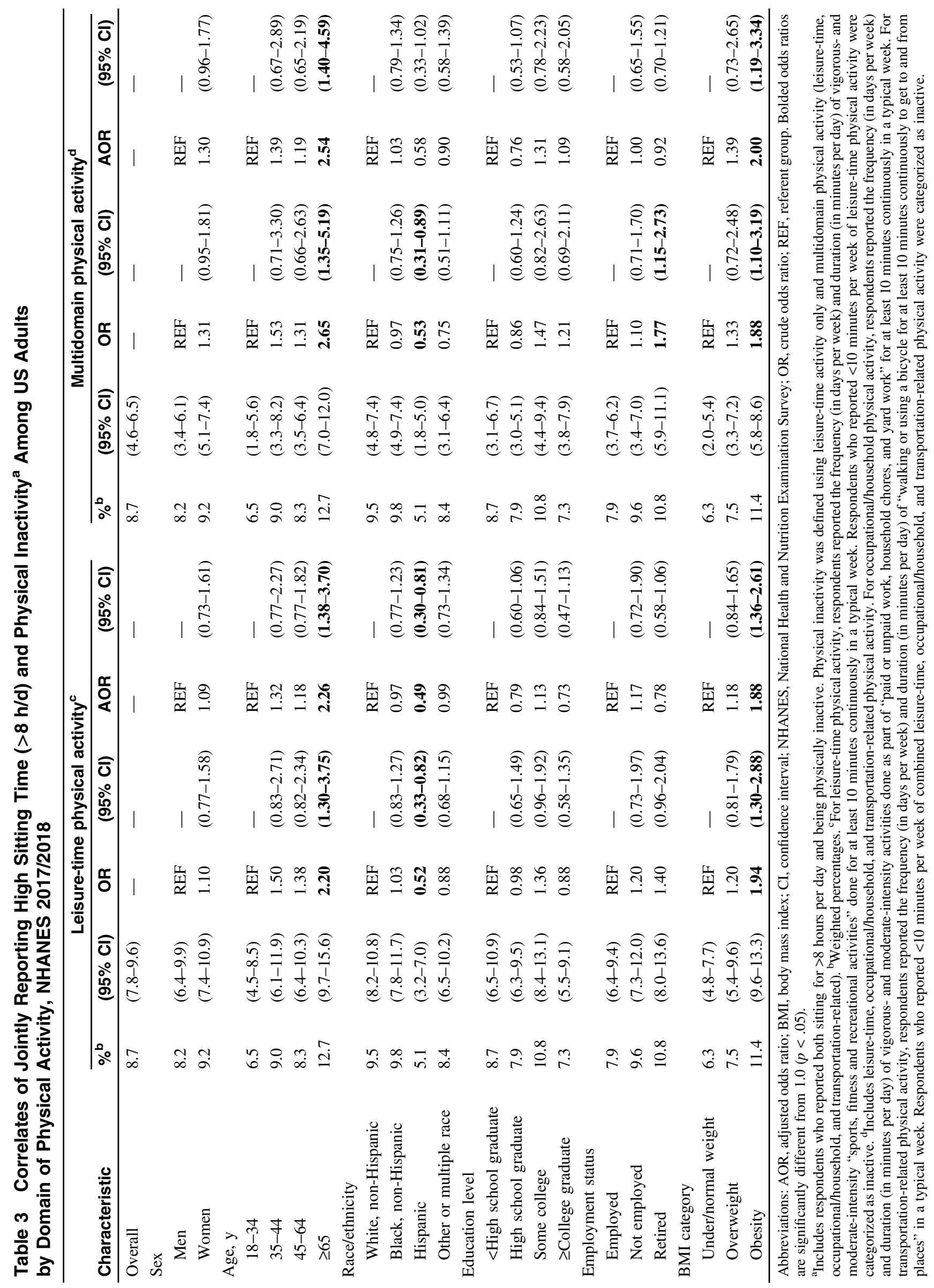


overall sitting time but does not separate sitting time into various domains (eg, work and leisure time). Population surveillance that quantifies the amount of time US adults spend being sedentary in specific contexts would help to inform domain-specific interventions to reduce sitting time.

Although studies investigating correlates of combined high sitting time and physical inactivity are limited, our findings are generally consistent with previous research that has separately established correlates of high sedentary behavior and low physical activity. ${ }^{10-14}$ Regardless of the physical activity measure, we found a positive association with older age (ages $\geq 65$ y old compared with 18-34 y) and higher BMI (adults with obesity compared with under/ normal weight). Multiple studies have found higher sedentary behavior (in isolation), as well as lower physical activity (in isolation), among older adults compared with younger adults and adults who are overweight or have obesity compared with normal weight adults. ${ }^{10-14}$ We also found that, compared with white adults, Hispanic adults had lower odds of being both high sitters and physically inactive. Although the existing literature shows that Hispanic adults have lower physical activity levels compared with white adults, $8,17,18$ studies have also demonstrated lower sedentary behavior among Hispanic adults ${ }^{6}$; thus, in the present study, the low joint prevalence in this group is likely to be driven by lower sitting time.

Overall, we found that the joint prevalence of high sitting time and multidomain physical inactivity was relatively low and declined since 2013/2014. These are promising findings, as this combination represents the group at highest risk of cardiovascular disease and premature all-cause mortality. ${ }^{2,3,15}$ Still, studies have shown that a high volume of physical activity is needed to ameliorate the adverse effects of high sitting time, ${ }^{2,3,15}$ and our findings suggest that strategies to increase physical activity levels, reduce sitting time, or a combination of both may be particularly important among adults who are older or have obesity. ${ }^{19}$ Moreover, recent small-scale studies have found that sedentary behavior has increased and physical activity has decreased during the COVID19 pandemic, further reinforcing the importance and timeliness of strategies to improve these behaviors. ${ }^{20}$

This study is subject to at least 2 limitations. First, estimates of sitting time and physical activity were based on self-reported data, which may be subject to measurement error. ${ }^{21,22}$ For example, sedentary behavior that is self-reported using a single-item measure has been shown to be underestimated when compared with accelerometer-derived estimates, ${ }^{14,21}$ suggesting that the estimates reported here may not reflect the true population levels of sitting time. Second, the question used to assess sedentary behavior in 2007/ 2008 was changed slightly in subsequent cycles to exclude time spent reclining. It is uncertain how this change in the question might have affected observed trends. The strengths of this study include the use of a nationally representative sample of adults and the use of a survey that assesses multiple domains of physical activity.

\section{Conclusions}

Since 2007/2008, daily sitting time has increased overall among US adults, although more recent data points suggest sitting has decreased since 2013/2014, as has the joint prevalence of high sitting and inactivity. Similar trends were observed among inactive, insufficiently active, and highly active adults, regardless of whether leisure time only or multidomain physical activity was considered. Nationwide efforts can help ensure that the promising decline in sitting time continues. Older adults and adults with obesity may especially benefit from interventions designed to help adults reduce the time spent sitting and incorporate moderate- to vigorousintensity activities into their daily routines.

\section{Acknowledgments}

No funding was secured for this study. The authors thank Michael Weeks, technical writer-editor, for manuscript editing. The findings and conclusions in this report are those of the authors and do not necessarily represent the official position of the Centers for Disease Control and Prevention or the National Institutes of Health.

\section{References}

1. U.S. Department of Health and Human Services. Physical Activity Guidelines for Americans. 2nd ed. Washington, DC: U.S. Department of Health and Human Services; 2018.

2. 2018 Physical Activity Guidelines Advisory Committee. Physical Activity Guidelines Advisory Committee Scientific Report. Washington, DC: U.S. Department of Health and Human Services; 2018.

3. Ekelund U, Steene-Johannessen J, Brown WJ, et al. Does physical activity attenuate, or even eliminate, the detrimental association of sitting time with mortality? A harmonised meta-analysis of data from more than 1 million men and women. Lancet. 2016;388(10051):1302-1310. PubMed ID: 27475271 doi:10.1016/S0140-6736(16)30370-1.

4. Omura JD, Whitfield GP, Chen TJ, et al. Surveillance of physical activity and sedentary behavior among youth and adults in the United States: History and opportunities. J Phys Act Health. 2021;18(suppl): S6-S24. doi:10.1123/jpah.2021-0179

5. Du Y, Liu B, Sun Y, Snetselaar LG, Wallace RB, Bao W. Trends in adherence to the Physical Activity Guidelines for Americans for aerobic activity and time spent on sedentary behavior among US adults, 2007 to 2016. JAMA Netw Open. 2019;2(7):e197597. PubMed ID: 31348504 doi:10.1001/jamanetworkopen.2019.7597

6. Yang L, Cao C, Kantor ED, et al. Trends in sedentary behavior among the US population, 2001-2016. JAMA. 2019;321(16):1587-1597. PubMed ID: 31012934 doi:10.1001/jama.2019.3636.

7. Whitfield GP, Ussery EN, Saint-Maurice PF, Carlson SA. Trends in aerobic physical activity participation across multiple domains among US Adults, National Health and Nutrition Examination Survey 2007/ 2008 to 2017/2018. J Phys Act Health. 2021;18(suppl):S64-S73. doi:10.1123/jpah.2021-0173

8. Whitfield GP, Hyde ET, Carlson SA. Participation in leisure-time aerobic physical activity among adults, National Health Interview Survey, 1998-2018. J Phys Act Health. 2021;18(suppl):S25-S36. doi:10.1123/jpah.2021-0014

9. Ussery EN, Fulton JE, Galuska DA, Katzmarzyk PT, Carlson SA. Joint prevalence of sitting time and leisure-time physical activity among US adults, 2015-2016. JAMA. 2018;320(19):2036-2038. PubMed ID: 30458482 doi:10.1001/jama.2018.17797.

10. Healy GN, Clark BK, Winkler EAH, Gardiner PA, Brown WJ, Matthews CE. Measurement of adults' sedentary time in population-based studies. Am J Prev Med. 2011;41(2):216-227. PubMed ID: 21767730 doi:10.1016/j.amepre.2011.05.005.

11. Harrington DM, Barreira TV, Staiano AE, Katzmarzyk PT. The descriptive epidemiology of sitting among US adults, NHANES 2009/2010. J Sci Med Sport. 2014;17(4):371-375. PubMed ID: 23988785 doi:10.1016/j.jsams.2013.07.017.

12. Bauman AE, Reis RS, Sallis JF, Wells JC, Loos RJF, Martin BW. Correlates of physical activity: why are some people physically active and others not? Lancet. 2012;380(9838):258-271. PubMed ID: 22818938 doi:10.1016/S0140-6736(12)60735-1. 
13. Prince SA, Reed JL, McFetridge C, Tremblay MS, Reid RD. Correlates of sedentary behaviour in adults: a systematic review. Obes Rev. 2017;18(8):915-935. PubMed ID: 28524615 doi:10.1111/obr.12529.

14. Bauman AE, Petersen CB, Blond K, Rangul V, Hardy LL. The descriptive epidemiology of sedentary behaviour. In: Leitzmann MF, Jochem C, Schmid D, eds. Sedentary Behaviour Epidemiology. Cham, Switzerland: Springer International Publishing; 2018:73-106.

15. Stamatakis E, Gale J, Bauman A, Ekelund U, Hamer M, Ding D. Sitting time, physical activity, and risk of mortality in adults. $J$ Am Coll Cardiol. 2019;73(16):2062-2072. PubMed ID: 31023430 doi:10.1016/j.jacc.2019.02.031.

16. NHLBI Obesity Education Initiative Expert Panel on the Identification, Evaluation, and Treatment of Obesity in Adults. Clinical Guidelines on the Identification, Evaluation, and Treatment of Overweight and Obesity in Adults: The Evidence Report. Bethesda, MD: National Heart, Lung, and Blood Institute; 1998.

17. Whitfield GP, Ussery EN, Carlson SA. Combining data from assessments of leisure, occupational, household, and transportation physical activity among US adults, NHANES 2011-2016. Prev Chronic Dis. 2020;17:E117. PubMed ID: 33006543 doi:10.5888/pcd17.200137
18. Moore LV, Harris CD, Carlson SA, Kruger J, Fulton JE. Trends in no leisure-time physical activity-United States, 1988-2010. Res $Q$ Exerc Sport. 2012;83(4):587-591. PubMed ID: 23367822 doi:10. 1080/02701367.2012.10599884.

19. Matthews CE. Minimizing risk associated with sedentary behavior: should we focus on physical activity, sitting, or both? J Am Coll Cardiol. 2019;73(16):2073-2075. PubMed ID: 31023431 doi:10. 1016/j.jacc.2019.02.030.

20. Meyer J, McDowell C, Lansing J, et al. Changes in physical activity and sedentary behavior in response to COVID-19 and their associations with mental health in 3052 US adults. Int $J$ Environ Res Public Health. 2020;17(18):6469. doi:10.3390/ijerph 17186469

21. Prince SA, Cardilli L, Reed JL, et al. A comparison of self-reported and device measured sedentary behaviour in adults: a systematic review and meta-analysis. Int J Behav Nutr Phys Act. 2020;17(1):31. PubMed ID: 32131845 doi:10.1186/s12966-020-00938-3

22. Sallis JF, Saelens BE. Assessment of physical activity by self-report: status, limitations, and future directions. Res Q Exerc Sport. 2000; 71(suppl 2):1-14. doi:10.1080/02701367.2000.11082780. 